\title{
Correction \\ Correction: Bates et al. Vector Fields and Differential Forms on the Orbit Space of a Proper Action. Axioms 2021, 10, 118
}

\author{
Larry Bates, Richard Cushman * and Jędrzej Śniatycki \\ Department of Mathematics and Statistics, University of Calgary, Calgary, AB T2N 1N4, Canada; \\ bates@ucalgary.ca (L.B.); sniatycki@gmail.com (J.Ś.) \\ * Correspondence: r.h.cushman@gmail.com
}

check for

updates

Citation: Bates, L.; Cushman, R.; Śniatycki, J. Correction: Bates et al. Vector Fields and Differential Forms on the Orbit Space of a Proper Action Axioms 2021, 10, 118. Axioms 2021, 10, 173. https://doi.org/10.3390/ axioms10030173

Received: 7 July 2021

Accepted: 27 July 2021

Published: 30 July 2021

Publisher's Note: MDPI stays neutral with regard to jurisdictional claims in published maps and institutional affiliations.

Copyright: (C) 2021 by the authors. Licensee MDPI, Basel, Switzerland. This article is an open access article distributed under the terms and conditions of the Creative Commons Attribution (CC BY) license (https:/ / creativecommons.org/licenses/by/ $4.0 /)$.
There was an error in the original article [1]. In the proof of lemma 5, Equation (19) is incorrect. A correction has been made to the proof of Lemma 5 in Section 2 Vector fields on $M / G$ to the fifth, sixth and seventh paragraphs.

The corrected fifth paragraph is:

The module $\mathfrak{X}(B)^{H}$ of $H$ invariant smooth vector fields on $B$ is finitely generated by polynomial vector fields, see [16], and we denote a generating set by $\left\{X_{j}\right\}_{j=1}^{N}$. Hence, every $H$ invariant smooth vector field $X_{B}$ on $B$ is of the form $X_{B}=\sum_{j=1}^{N} h_{j} X_{j}$ for some $h_{1}, \ldots, h_{n} \in C^{\infty}(B)$. Similarly, every $K$ invariant smooth vector field on $B_{(K)}$ can be written as $\sum_{j=1}^{N} f_{j} X_{j}$, where $f_{j} \in C^{\infty}\left(B_{(K)}\right)$. Since $B_{(K)}$ is open and dense in $B$, a generic $f \in C^{\infty}\left(B_{(K)}\right)$ need not extend to a smooth function on $B$. Therefore a generic vector field on $B_{(K)}$ need not extend to a smooth vector field on $B$. On the other hand, the $H$ invariant vector field $X_{B_{(K)}}$ on $B_{(K)}$, is obtained above from a smooth bounded vector field $Y$ on $B / H$. Therefore

$$
X_{B_{(K)}}(\mathbf{x})=\sum_{j=1}^{N} k_{j \mid B_{(K)}}(\mathbf{x}) X_{j}(\mathbf{x}),
$$

for each $\mathbf{x} \in B_{(K)} \subseteq B \subseteq \mathbb{R}^{n}$, where every $k_{j} \in C^{\infty}\left(\mathbb{R}^{n}\right)$, and $k_{j \mid B_{(K)}}$ is the restriction of $k_{j}$ to $B_{(K)}$.

Since $B_{(K)}$ is open and dense in $B$, we may define

$$
X(\mathbf{x})=\left\{\begin{array}{cl}
X_{B_{(K)}}(\mathbf{x}) & \text { if } \mathbf{x} \in B_{(K)} \\
\sum_{i=1}^{N} \lim _{k \rightarrow \infty} k_{j}\left(\mathbf{x}_{k}\right) X_{j}\left(\mathbf{x}_{k}\right), & \text { where }\left\{\mathbf{x}_{k}\right\} \subseteq B_{(K)} \text { and } \\
& \mathbf{x}=\lim _{k \rightarrow \infty} \mathbf{x}_{k} \in B \backslash B_{(K)},
\end{array}\right.
$$

provided that $\lim _{k \rightarrow \infty} k_{j}\left(\mathbf{x}_{k}\right)$ exists and is unique. Since the vector fields $X_{j}\left(\mathbf{x}_{k}\right)$ are smooth on $B$,

$$
\lim _{k \rightarrow \infty} k_{j}\left(\mathbf{x}_{k}\right) X_{j}\left(\mathbf{x}_{k}\right)=\left(\lim _{k \rightarrow \infty} k_{j}\left(\mathbf{x}_{k}\right)\right) X_{j}\left(\lim _{k \rightarrow \infty} \mathbf{x}_{k}\right) \text { for every } j=1, \ldots, N .
$$

The corrected sixth paragraph is:

Moreover, since $B_{(K)}$ is open and dense in $B$, it is open and dense in $\bar{B}$, the closure of $B$. In Equation (19), each function $k_{j \mid B_{(K)}}$ is the restriction to $B_{(K)} \subseteq B \subseteq \mathbb{R}^{n}$ of a smooth function $k_{j}$ on $\mathbb{R}^{n}$. Moreover, the choice of polynomial basis $\left\{X_{j}\right\}_{j=1}^{N}$ ensures that the right-hand side of Equation (19) extends to the closure $\bar{B}$ of $B$. Hence all the the limits $\lim _{k \rightarrow \infty} k_{j}\left(x_{k}\right)$ in Equation (20) exist, and $X(\mathbf{x})$ is defined for all $\mathbf{x} \in B$.

The corrected seventh paragraph is:

We need to show that this definition of $X$ on $B$ depends only on $X_{B_{(K)}}$. Since each $k_{j}$ is continuous on $B$ and its first partial derivatives are bounded on $\bar{B}$, it follows that $k_{j}$ are uniformly continuous on $\bar{B}$. In particular, if $c:[0,1] \rightarrow \bar{B}$ is a smooth curve, such that $c([0,1)) \subseteq B_{(K)}$ and $c(1) \in B$, then 


$$
k_{j \mid B}(c(1))=k_{j \mid B}(c(0))+\int_{0}^{1} \frac{\partial k_{j \mid B}}{\partial t}(c(t)) d t=k_{j \mid B_{(K)}}(c(0))+\int_{0}^{1} \frac{\partial k_{j \mid B_{(K)}}}{\partial t}(c(t)) d t .
$$

Thus, the values of $k_{j}$ on $B$ are uniquely determined by $k_{j \mid B_{(K)}}$. Repeating this argument for all the first-order partial derivatives of $k_{j}$, we deduce that the first-order partial derivatives of $k_{j}$ on $B$ are uniquely determined by $k_{j \mid B_{(K)}}$ and its first partial derivatives. Continuing this process for every partial derivative of every order shows that the restriction of $k_{j}$ to $B$ is uniquely determined by $k_{j \mid B_{(K)}}$.

The authors apologize for any inconvenience caused and state that the scientific conclusions are unaffected. The original article has been updated.

Acknowledgments: The authors would like to thank Editor Luna Shen for her invitation to publish a feature paper in Axioms. The authors are grateful to Gerald Schwarz for pointing out an error in the proof of Lemma 5, and for suggesting the inclusion in the bibliography of three additional papers [2-4], related to the problem under consideration.

\section{References}

1. Bates, L.; Cushman, R.; Śniatycki, J. Vector Fields and Differential Forms on the Orbit Space of a Proper Action. Axioms 2021, 10, 118. [CrossRef].

2. Schwarz, G. Lifting smooth homotopies of orbit spaces. IHES Pub. Math. 1980, 51, 37-135.

3. Kankaanrinta, M. Lifting smooth homotopies of orbit spaces of proper Lie group actions. J. Lie Theory 2005, 15, 447-456.

4. Karshon, Y.; Watts, J. Basic forms and orbit spaces: A diffeological approach. SIGMA Symmetry Integr. Geom. Methods Appl. 2016, $12,19$. 